\title{
Eimeria vermiformis Infection Model of Murine Small Intestine
}

Patrícia Figueiredo-Campos\#, Cristina Ferreira\#, Birte Blankenhaus and Marc Veldhoen*

Instituto de Medicina Molecular | João Lobo Antunes, Faculdade de Medicina, Universidade de Lisboa, Lisbon, Portugal

*For correspondence: marc.veldhoen@medicina.ulisboa.pt

\#Contributed equally to this work

\begin{abstract}
[Abstract] Eimeria vermiformis is a tissue specific, intracellular protozoan that infects the murine small intestinal epithelia, which has been widely used as a coccidian model to study mucosal immunology. This mouse infection model is valuable to investigate the mechanisms of host protection against primary and secondary infection in the small intestine. Here, we describe the generation of an E. vermiformis stock solution, preparation of sporulated $E$. vermiformis to infect mice and determination of oocysts burden. This protocol should help to establish a highly reproducible natural infection challenge model to study immunity in the small intestine. The information obtained from using this mouse model can reveal fundamental mechanisms of interaction between the pathogen and the immune response, e.g., provided by intraepithelial lymphocytes (IEL) at the basolateral site of epithelial cells but also a variety of other immune cell populations present in the gut.
\end{abstract}

Keywords: Eimeria vermiformis, Small intestine, Infection model, Coccidiosis, Apicomplexan, Mucosal immunology

[Background] Infections of the small intestine are widespread and can cause significant morbidity and mortality worldwide (Munot and Kotler, 2016). Children and the elderly, due to their weaker immune system, suffer the worst consequences. Gaining a better understanding of how the small intestinal immune system functions is therefore of prime importance. However, many of the pathogens used in mouse models are not natural to the host, can cause systemic inflammation or can persist in the tissues for long periods. This can result in uncharacteristic induction of the immune response and inadequate development of protective immunity.

Eimeria vermiformis is a tissue specific, intracellular single cell protozoan, which infects the small intestinal epithelia. Unlike the related pathogen used in infection models, Toxoplasma gondii, this is a natural, self-limiting, murine parasite, which induces a strong immune response and establishes longlasting protection against subsequent infections. Furthermore, E. vermiformis is monoxenous, its life cycle is completed without the need of a second host species (see Figure 1). It is therefore an ideal pathogen to study the mechanisms of host protection against primary and secondary infection at the small intestinal barrier.

Mice infected with E. vermiformis excrete a non-infectious, unsporulated form of oocysts into the environment. Once in contact with oxygen and moisture, in a process which takes between 2 to 7 days, these oocysts mature into an infectious, sporulated form, which, upon ingestion, disseminates the 
infection to other animals (Fayer, 1980).

The $E$. vermiformis infection model has been used in the past and has yielded valuable information in areas such as the role of $\alpha \beta$ and $\gamma \delta$ T cells that line the small intestinal epithelia (Roberts et al., 1996) and the influence of age of the host in the development of protective immune responses in the intestine (Ramsburg et al., 2003). More recently we have used the E. vermiformis model to highlight the role of mitochondria in controlling the activation state of epithelial-resident T lymphocytes (Konjar et al., 2018).

Here, we present an adapted version of the original protocol used by Long et al. (1976) to purify and sporulate E. vermiformis. We describe in detail the method used to propagate the parasite, infect mice and accurately assess parasite load.

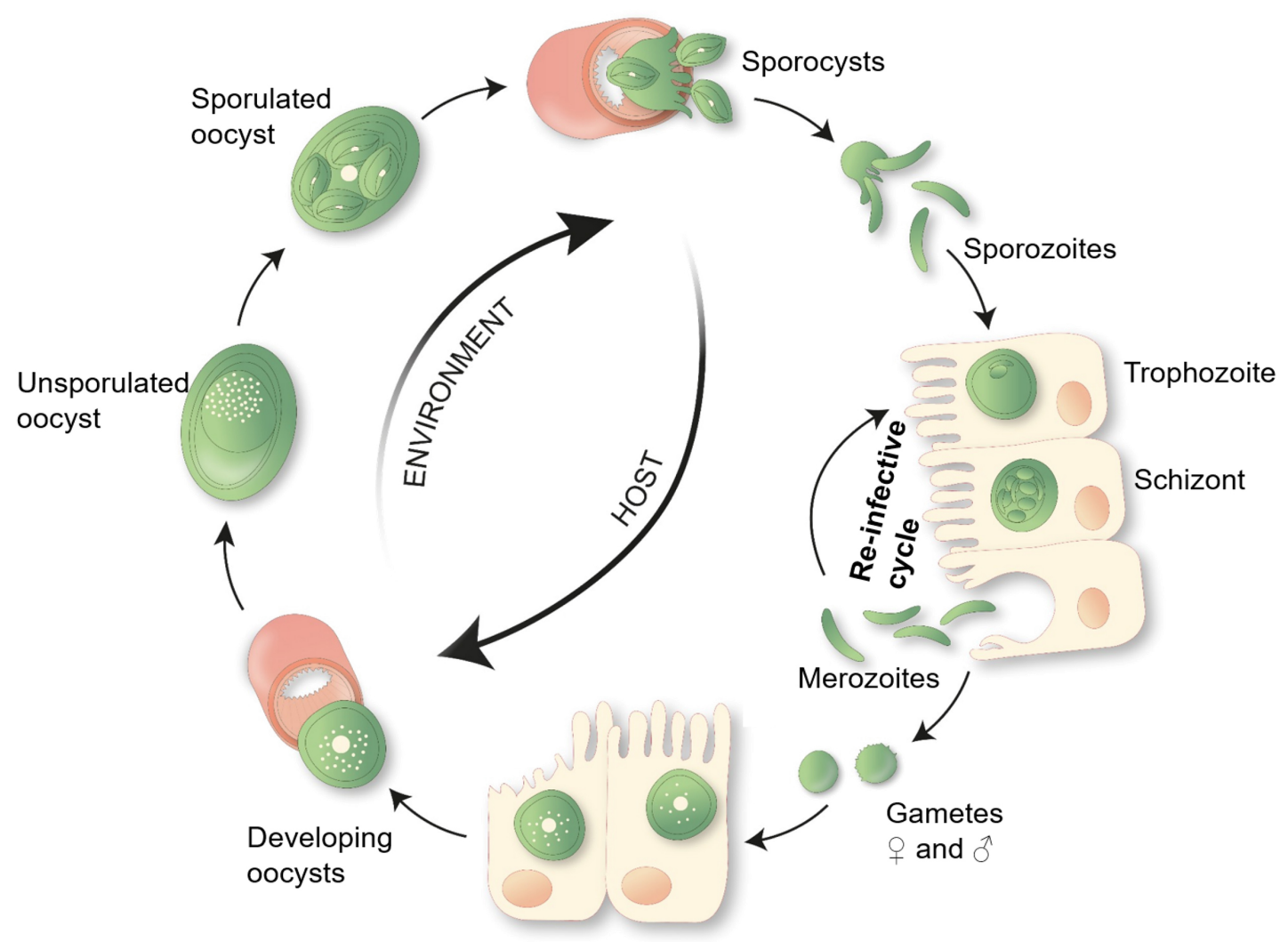

Figure 1. E. vermiformis life cycle. A single sporulated oocyst can initiate an infection when swallowed by its respective host, the mouse. Each mature oocyst holds four sporocysts, each sporocyst gives rise to two sporozoites, which can infect the enterocytes. Inside the host cell, the parasite develops via trophozoite and schizont stages into merozoites. Merozoites can re-infect the epithelial cells and start a new cycle. Eimeria typically undergoes three rounds of asexual multiplication whereupon merozoites give rise to gametes. Male and female gametes can fuse to form an oocyst, protected by a multi-layered cell wall making them highly resistant to environmental pressures. These oocysts shed with the feces. Unsporulated oocysts undergo sporulation upon contact with oxygen and moisture, undergoing meiosis, which takes between 2 to 7 days. 


\section{Materials and Reagents}

1. Millex ${ }^{\circledR}$-GP Filter Unit: Millipore Express ${ }^{\circledR}$ PES membrane $0.22 \mu \mathrm{m}$ (Merk Millipore Ltd., catalog number: SLGP033RS)

2. Fine mesh metal sieve (no specific brand) $(2 x)$

3. Aluminum foil (no specific brand)

4. $50 \mathrm{ml}$ centrifuge tube, CentriStar ${ }^{\mathrm{TM}}$ cap, polypropylene, RNase-/DNase-free, Nonpyrogenic (Corning Science Mexico, catalog number: 430921)

5. $15 \mathrm{ml}$ centrifuge tube, CentriStar ${ }^{\mathrm{TM}}$ cap, polypropylene, RNase-/DNase-free, Nonpyrogenic (Corning Science Mexico, catalog number: 430791)

6. $2 \mathrm{ml}$ microcentrifuge tube graduated natural RNase-/DNase-free (Fisher Scientific, catalog number: 05-408-138)

7. $1,000 \mu \mathrm{l}$ Pipette tips (no specific brand)

8. $200 \mu$ l Pipette tips (no specific brand)

9. Fine sand, e.g., for playground and sand pits (Sand can be autoclaved for use in biological containment facilities)

10. C57BL6/J mouse strain and/or strain and lines of interest

11. Eimeria vermiformis (not commercially available)

12. Sterile tap water

13. Sodium hypochlorite $14 \%$ C12 in aqueous solution (VWR CHEMICALS, catalog number: 27900.365) (Room temperature)

14. Sodium chloride ( $\mathrm{NaCl}$ ) (Merck KGaA, catalog number: 1. 06404.1000) (Room temperature)

15. Potassium dichromate $\left(\mathrm{Cr}_{2} \mathrm{~K}_{2} \mathrm{O}_{2}\right)(\mathrm{VWR}$, catalog number: 26784.297)

16. Fridge (no specific brand)

\section{Equipment}

1. Fuchs-Rosenthal Counting Chamber (MARIENFELD, catalog number: 0640410)

2. Two-chamber McMaster Counting Chamber (CHALEX, LLC: www.vetslides.com)

3. Funnel (no specific brand)

4. $1 \mathrm{~L}$ Beaker (no specific brand)

5. P1000 Pipette (no specific brand)

6. P200 Pipette (no specific brand)

7. Magnetic stirrer (VELP Scientifica, model: F20500162)

8. Fume hood (Burdinola, model: OR-ST-1500)

9. Aquarium pump (no specific brand, e.g., $1 \mathrm{~W}$ silent pump)

10. Centrifuge (Eppendorf AG, 22331 Hamburg, model: 5810 R)

11. Grant-Bio Vortex, PV-1 (Grant Instruments Ltd)

12. Microscope (Nikon, Japan, model: SE) 
13. Inverted microscope (EU importer-Carl Zeiss Microscopy $\mathrm{GmbH}$, model: Primovert, catalog number: 415519-1100-000)

\section{Procedure}

A. Preparing E. vermiformis stock

1. Infect mice with $E$. vermiformis (1,000 oocysts) in $100 \mu \mathrm{l}$ of tap-filtered water via oral gavage.

2. At Day 8 post-infection, place the mice in a cage lined with sand (refer to Procedure $B$ and Figure 3).

Note: Susceptibility to and patency of infection is mouse strain dependent and should be empirically tested. The protocol described here is typical for the C57BL6/J mouse strain.

3. Collect mouse feces from a cage containing five infected animals, on Day 9 post-infection.

4. Place feces into a $50 \mathrm{ml}$ Falcon tube, add $40 \mathrm{ml}$ of sterile water and vortex until feces disaggregate. Store in a fridge $\left(2-6^{\circ} \mathrm{C}\right)$.

Note: Use of harsher methods such as glass beads may damage the parasites.

5. Repeat procedure, Steps A3-A4, on Days 10 and 11 post-infection.

6. When ready to prepare the stock, pour the contents of the three Falcon tubes through a fine mesh sieve, into a beaker (to remove larger pieces).

7. Working in a fume hood, add $180 \mathrm{ml}$ of a $4 \%$ potassium dichromate solution $(\mathrm{w} / \mathrm{v})$, making up a final concentration of $2.4 \%(3 \times 40 \mathrm{ml}+180 \mathrm{ml}=300 \mathrm{ml}$ end volume $)$.

8. Add a stir bar to the beaker and place on a magnetic stirrer. Aerate the contents using an aquarium pump on a low setting, enough to create a few air bubbles continuously.

9. Cover beaker with aluminum foil to reduce evaporation.

10. Leave the pump on and keep stirring at room temperature in the fume hood for three days. Regularly check stirrer and aeration.

11. After three days, take a sample and count oocysts in a Fuchs Rosenthal Chamber to check the percentage of sporulation (refer to Procedure $\mathrm{C}$ and Figure 2). If sporulation is under $70 \%$, leave the solution for another day and repeat the counting and sporulation assessment the next day or the day after that if required (generally takes 4-5 days).

12. Once the percentage of sporulation is higher than $70 \%$, place the stock solution in the fridge. Note down the approximate concentration of oocysts for future reference. 

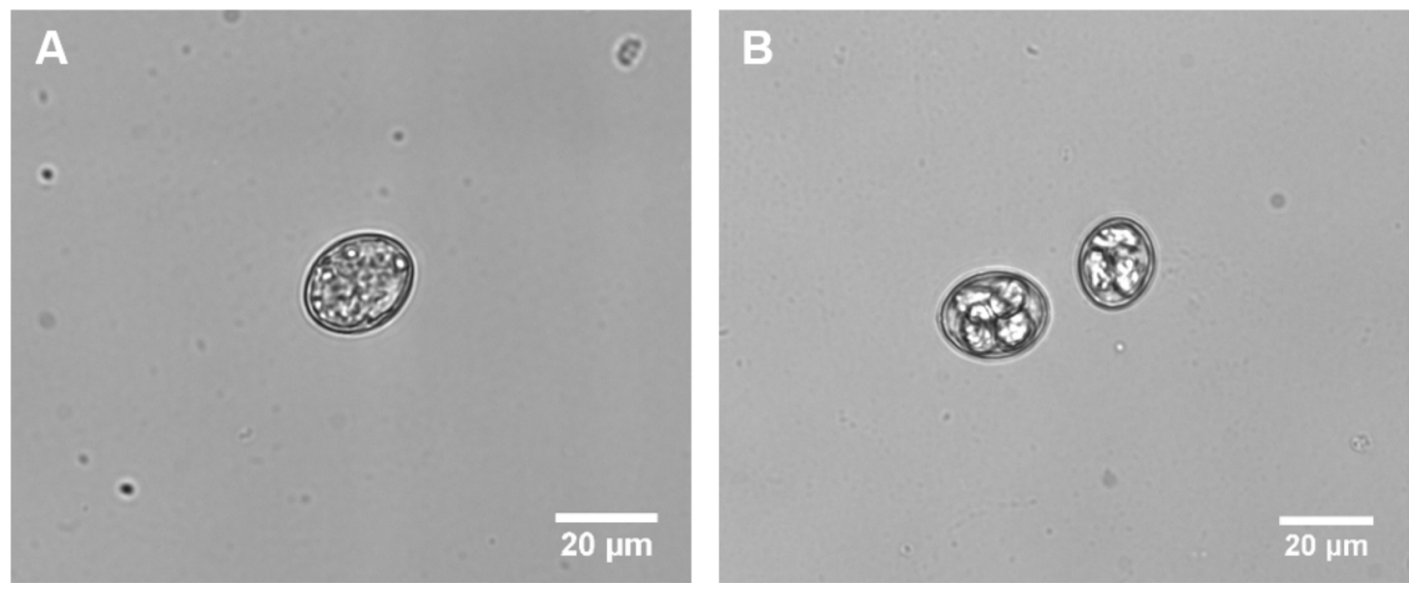

Figure 2. E. vermiformis oocysts. This figure illustrates how "to distinguish sporulated oocyst". A. Unsporolated or early oocyst. B. Sporulated or late oocyst, which is tetrasporic in which four sporocysts are visible. Recorded at Primovert microscope, taken at 400x magnification (Ocular $10 x+$ objective $40 x)$. Scale bar $=20 \mu \mathrm{m}$.

B. Preparing E. vermiformis to infect mice

1. Transfer an appropriate volume containing oocysts from the E. vermiformis stock solution (refer to Procedure A) into a $15 \mathrm{ml}$ Falcon tube.

Notes:

a. Usually, stock solution contains approximately $1 \times 10^{5}$ oocysts $/ \mathrm{ml}$.

b. Appropriate volume depends on how many mice you wish to infect and the stock solution obtained.

2. Fill up to $15 \mathrm{ml}$ with sterile water and centrifuge for $8 \mathrm{~min}$ at $1,800 \times g$ at room temperature.

3. Discard the supernatant and vortex to detach the pellet from the bottom of the Falcon tube.

4. Fill up again to $15 \mathrm{ml}$ with sterile water and repeat the washing step twice more.

Note: Typically, the supernatant changes from light yellow to clear.

5. Discard the supernatant, vortex the pellet and fill up with Sodium Hypochlorite to $15 \mathrm{ml}$.

Note: It is important to thoroughly re-suspend the pellet in the Sodium Hypochlorite since the oocysts are at the bottom and need to float up, to the top of the Sodium Hypochlorite. Use a vortex to mix.

6. Centrifuge for $10 \mathrm{~min}$ at $1,100 \times g$ with low break (setting 1 ), room temperature. Take the tube very carefully from the centrifuge and cautiously remove $1-2 \mathrm{ml}$ from the surface ring and transfer to a clean $15 \mathrm{ml}$ Falcon tube.

Note: For the top 1-2 ml, you may spot a slight cloudiness by eye.

7. Optional: After removing the top 1-2 ml (Step B6), fill up again with Sodium Hypochlorite, vortex to re-suspend the pellet and repeat the centrifugation for $10 \mathrm{~min}$, at 1,100 $\times \mathrm{g}$, low break, at room temperature. Add $1 \mathrm{ml}$ from the surface ring to the previously collected $1-2 \mathrm{ml}$ in the $15 \mathrm{ml}$ Falcon tube.

8. Fill up the tube containing $2-3 \mathrm{ml}$ of oocysts to $15 \mathrm{ml}$ with sterile water and centrifuge for $8 \mathrm{~min}$ 
at $1,800 \times g$, at room temperature.

9. Discard the supernatant and fill up again to $15 \mathrm{ml}$ with sterile water. Repeat the washing step twice more.

10. In the last washing step, discard the supernatant and re-suspend the pellet in $1 \mathrm{ml}$ of sterile filtered tap water.

C. Counting sporulated oocysts to infect mice

1. Dilute the previously prepared E. vermiformis to count in a Fuchs Rosenthal Chamber $0.200 \mathrm{~mm}$ depth, using the $10 \mathrm{x}$ objective (Figure 3).

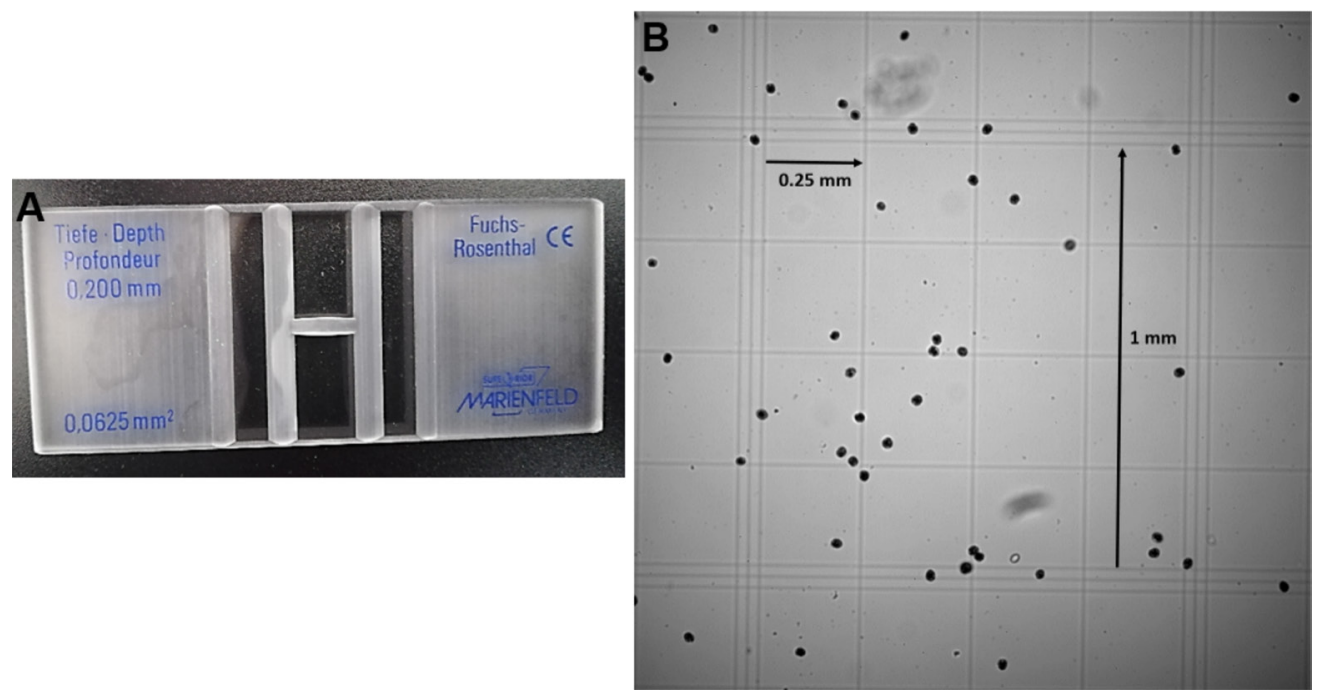

Figure 3. Fuchs Rosenthal Counting Chamber. A. Two-chamber counting slides $(0.200 \mathrm{~mm}$ depth; $0.0625 \mathrm{~mm}^{2}$ ). B. In total the grid contains 16 large squares of $1 \mathrm{~mm}$ side, enclosed by triple lines. All 16 large squares are subdivided into 16 small squares of $0.25 \mathrm{~mm}$ side. Due to the large counting grid and a depth of $0.2 \mathrm{~mm}$ the total volume amount is $3.2 \mu \mathrm{l}$, so the chamber factor is 312.5 .

2. Count sporulated oocysts in the 16 big squares and multiply by the chamber factor 312.5 $(0.2 \mathrm{~mm}$ height; volume of $3.2 \mu \mathrm{l})$ and the dilution to obtain number of sporulated oocysts $/ \mathrm{ml}$.

e.g., 60 sporulated oocysts $\times 312.5 \times 10$ (dilution 1:10) $=187,500$ oocysts $/ \mathrm{ml}$

$$
=1.875 \times 10^{5} \text { oocysts } / \mathrm{ml}
$$

Note: See Figure 2 for distinguishing sporulated and non-sporulated oocysts.

3. Adjust to the desired number of oocysts/ml. e.g., 1,000 oocysts/mouse in $100 \mu \mathrm{l}$. From the previous example, to prepare $1 \mathrm{ml}$ with the concentration of $1 \times 10^{4}$ oocysts $/ \mathrm{ml}$ :

$1,000 \mu \mathrm{l} / 1.875 \times 10^{5}$ oocysts $\times 10,000$ oocysts $=53.3 \mu \mathrm{l}$ from the prepared E. vermiformis solution $+946.7 \mu \mathrm{l}$ of sterile filtered tap water

Note: Prepare extra volume to account for the loss of volume in the gavage needle. The prepared E. vermiformis solution can be kept in the fridge overnight.

4. Give $100 \mu \mathrm{l}$ to each mouse via oral gavage (standard mouse size). 
D. Determining oocyst burden in mouse feces

1. On Day 6 post infection, place each mouse individually in a cage lined with fine sand (e.g., playground sand).

Note: Use a thin layer of sand, enough to cover the bottom of the cage (Figure 4).

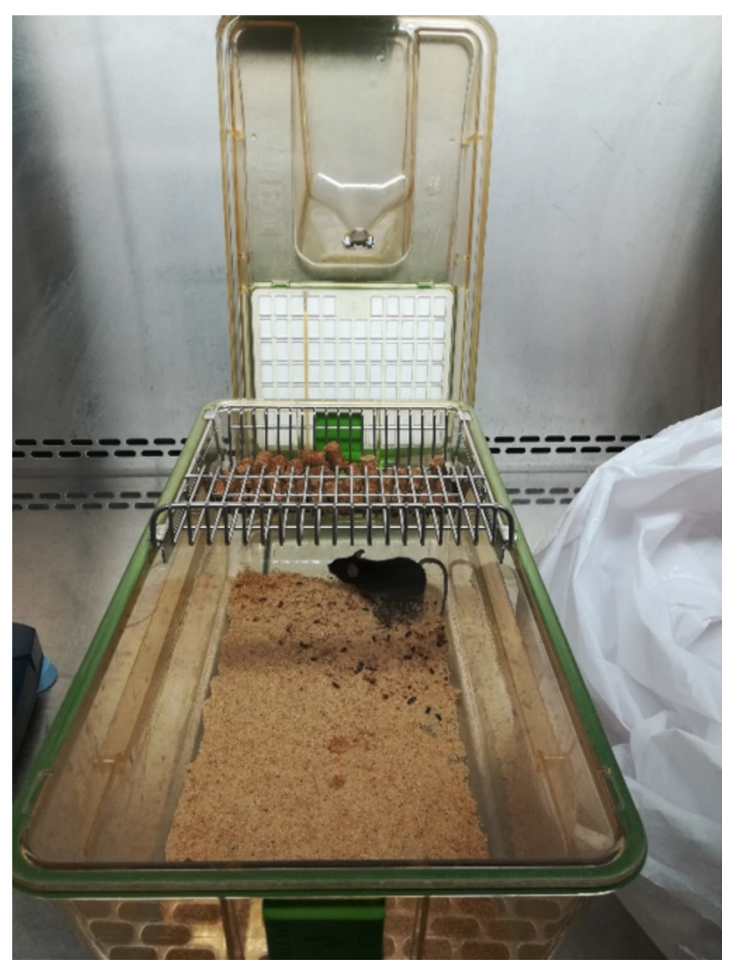

Figure 4. Mouse housed in Individually Ventilated Cage (IVC) lined with fine sand

2. On Day 7, collect the sand with feces from each mouse in each cage. Separate the sand from the feces using a sieve and transfer the feces into a $50 \mathrm{ml}$ Falcon tube using a funnel. Line the cage with clean sand and return the mouse to the cage.

3. Add $30 \mathrm{ml}$ of sterile water to the Falcon tube and vortex for $1 \mathrm{~min}$. Samples can be stored in the fridge for up to 6 months, prior to oocyst counting.

4. Repeat Steps C2 and C3 daily until no more oocysts are detected (15-16 days in C57BL6/J mice) for two consecutive days to determine patency (days of oocyst shedding) and accumulative number of oocysts.

5. Alternatively, if patency periods are similar between mouse lines or conditions tested and since the majority of oocysts are shed between Days 8-10, a shorter defined period may be chosen and reported.

E. Counting total oocysts in infected mouse feces

1. To count, vortex the $50 \mathrm{ml}$ Falcon tube containing the feces in $30 \mathrm{ml}$ volume of an individual mouse from one day vigorously to disaggregate the feces. 
2. Prepare 3 independent dilutions for each sample in saturated sodium chloride (final volume $1 \mathrm{ml}$, e.g., dilution 1:10-100 $\mu \mathrm{l}$ of feces $+900 \mu \mathrm{l}$ of sodium chloride).

Note: It is important to cut the tip end of the 200 and 1,000 $\mu$ tips (1 cm), in order to take up and transfer the fecal pieces from the sample.

3. Adjust the dilution to count up to 100 oocysts in each counting chamber.

Note: On the peak of infection higher oocysts numbers will be encountered. Prepare serial dilutions, not exceeding more than 3 dilution steps (refer to Table 1).

Table 1. Guide to dilute $E$. vermiformis in mouse feces to count in a McMaster Chamber. Dilutions are dependent on the day post-infection (d.p.i.). These dilutions are representative for C57BL6/J mice infected with 1,000 oocysts.

\begin{tabular}{|l|l|l|l|l|l|l|l|l|l|l|l|}
\hline d.p.i. & 7 & 8 & 9 & 10 & 11 & 12 & 13 & 14 & 15 & 16 & 17 \\
\hline Dilution & $1: 10$ & $1: 10$ & $1: 1,000$ & $1: 2,000$ & $1: 2,000$ & $1: 2,000$ & $1: 1,000$ & $1: 500$ & $1: 100$ & $1: 10$ & $1: 10$ \\
& & & & & & $1: 1,000$ & & & & & \\
\hline
\end{tabular}

4. Fill up the McMaster counting chamber (Figure 5) and wait $\sim 2$ min for the oocysts to float up in the saturated sodium chloride solution.

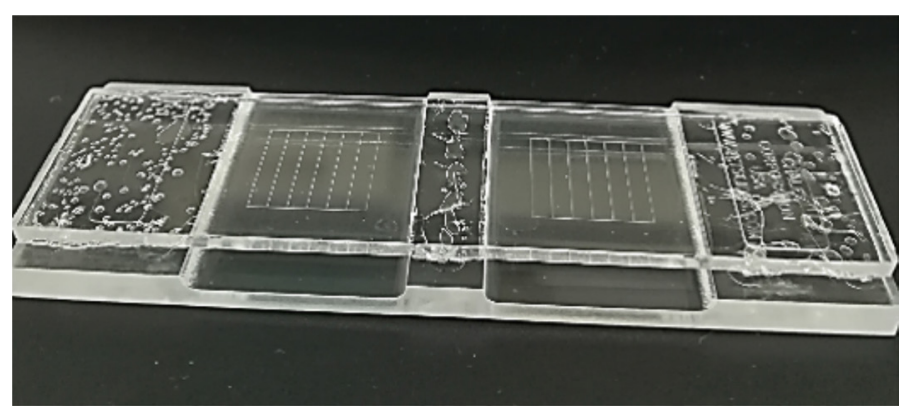

Figure 5. Two-chamber McMaster counting slide. $0.15 \mathrm{~cm}$ depth; $1 \mathrm{~cm}^{2}$.

5. Count total oocysts inside the grid areas using the 10x objective (include oocysts on the left and top grid lines).

6. Take the average of the triplicate counts and multiply by the dilution factor (chamber volume of $150 \mu \mathrm{l}$ and original volume of $30 \mathrm{ml}$; dilution factor is $200 \mathrm{x}$ ) as well as any additional dilutions used to obtain the total number of oocysts/mouse/day (Figure 6).

Formula: Average of oocysts counted from three chambers $x 200 \times$ additional dilution $=$ number of oocysts/mouse per day 


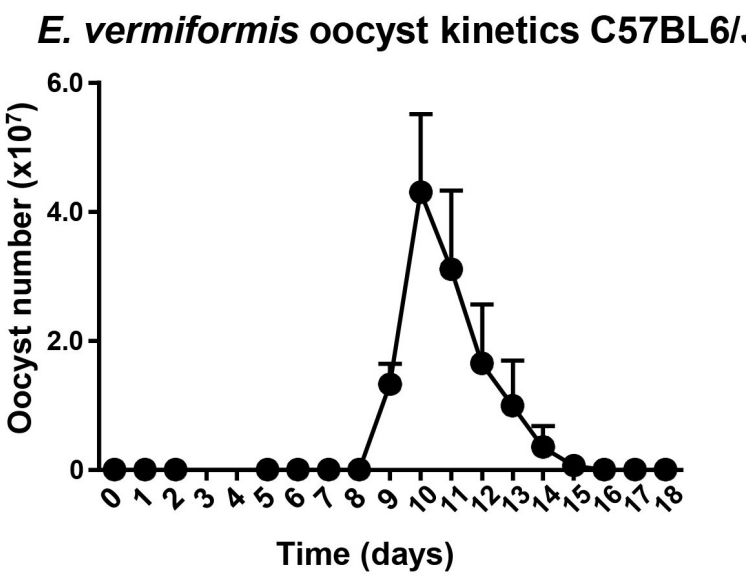

Figure 6. Characteristic oocyst burden of C57BL6/J mice. Counts of oocysts in the feces of mice inoculated with 1,000 sporulated oocysts of $E$. vermiformis. Three animals were analyzed per time point. Error bars show standard error of the mean (SEM).

\section{Acknowledgments}

This work was supported by the European Union H2020 ERA project (No. 667824EXCELLtoINNOV) for work in the Veldhoen laboratory and Fundação para a Ciência e a Tecnologia (FCT) Studentship SFRH/BD/131605/2017 to P.F-C. The authors like to thank Ana Figueiredo Duarte for designing Figure 1.

Application of the method described here can be found in reference Konjar et al. (2018).

\section{Competing interests}

The authors declare no competing financial interests.

\section{Ethics}

All animal experimentations complied with regulations of the Direção-Geral de Alimentação e Veterinária Portugal and local ethical review committee and guidelines at Instituto de Medicina Molecular | João Lobo Antunes.

\section{References}

1. Fayer, R. (1980). Epidemiology of protozoan infections: The coccidia. Vet Parasito/ 6(1): 75-103.

2. Konjar, Š., Frising, U. C., Ferreira, C., Hinterleitner, R., Mayassi, T., Zhang, Q., Blankenhaus, B., Haberman, N., Loo, Y., Guedes, J., Baptista, M., Innocentin, S., Stange, J., Strathdee, D., Jabri, B. and Veldhoen, M. (2018). Mitochondria maintain controlled activation state of epithelialresident T lymphocytes. Sci Immunol 3(24): eaan2543. 
3. Long, P. L., Millard, B. J., Joyner, L. P. and Norton, C. C. (1976). A guide to laboratory techniques used in the study and diagnosis of avian coccidiosis. Folia Vet Lat 6(3): 201-217.

4. Munot, K. and Kotler, D. P. (2016). Small Intestinal Infections. Curr Gastroenterol Rep 18(6): 31.

5. Ramsburg, E., Tigelaar, R., Craft, J. and Hayday, A. (2003). Age-dependent requirement for gammadelta $T$ cells in the primary but not secondary protective immune response against an intestinal parasite. J Exp Med 198(9): 1403-1414.

6. Roberts, S. J., Smith, A. L., West, A. B., Wen, L., Findly, R. C., Owen, M. J. and Hayday, A. C. (1996). T-cell alpha beta + and gamma delta + deficient mice display abnormal but distinct phenotypes toward a natural, widespread infection of the intestinal epithelium. Proc Natl Acad Sci U S A 93(21): 11774-11779. 